\title{
Immune System and Bone Microenvironment: Rationale for Targeted Cancer Therapies
}

Antonio Gnoni ${ }^{1}$, Oronzo Brunetti ${ }^{2} *$, Vito Longo $^{3}$, Angela Calabrese ${ }^{4}$, Antonella Argentiero ${ }^{1}$, Antonio Solimando ${ }^{5}$, Antonella Licchetta ${ }^{1}$.

1. Medical Oncology Unit, "S. Cuore di Gesù" Hospital, Gallipoli, Italy

2. Medical Oncology Unit, National Cancer Research Centre, IRCCS Istituto Tumori

"Giovanni Paolo II", Bari, Italy

3. Radiology Unit, National Cancer Research Centre, IRCCS Istituto Tumori "Giovanni Paolo II", Bari, Italy

4. Medical ThoracicOncology Unit, IRCCS Istituto Tumori "Giovanni Paolo II", Viale Orazio Flacco, 65, 70124 Bari, Italy

5. Department of Biomedical Sciences and Human Oncology, Section of Internal Medicine "G. Baccelli", University of Bari Medical School, Bari, Italy

*Corresponding Author

Brunetti Oronzo

dr.oronzo.brunetti@tiscali.it - 0805555914

MedicalOncology Unit

Istituto Tumori "Giovanni Paolo II

Viale Orazio Flacco, 65

70124 Bari

Italy 


\begin{abstract}
Osteoimmunology was coined about twenty years ago to identify a strict cross talk between bone niche and immune system both in physiological and pathological activities, including cancer. Several molecules are involved in the complex interaction between bone niche, immune and cancer cells. The Receptor Activator ok NF-kB (RANK)/RANK Ligand (RANKL/Osteoprotegerin (OPG) pathway plays a crucial role in bone cells/cancer interactions with subsequently immune system control failure, bone destruction, inhibition of effect and metastasis outcome. The bidirectional cross talk between bone and immune system could became a potential target for anticancer drugs. Several studies evidenced a direct anticancer role with improved survival of bone-targeted therapies such as bisphosphonates and RANKL antagonist Denosumab. Conversely, initial data evidenced a possible anti-bone resorption effect of systemic anticancer drugs through and immunomodulation activity, i.e. new generation antiandrogens (Abiraterone) in prostate cancer. All data could open a future rationale of combined bone, immunologic and targeted therapies in cancer treatment.
\end{abstract}

\title{
Keywords:
}

Antiandrogen, bisphosphonates, bone niche, immune system, osteoimmunology, RankL, targeted therapy.

\section{Index:}

1. Bidirectional crosstalk between Immune System and Bone niche: "osteoimmunology" concept

2. Alterations of Bone and Immune System in cancer: preclinical data

3. Osteoimmunology "balance" as potential target: first clinical data of a bidirectional anticancer activity with an immune-modulation

3.1 Bone targeted drugs have a role in anticancer systemic therapy through immune system activation

3.2 Anticancer drugs play effect against bone disruption through immunomodulation

4. Conclusion 


\section{Bidirectional crosstalk between Immune System and Bone niche: "osteoimmunology" concept}

Osteoimmunology was coined about twenty years ago to identify a new interdisciplinary field, between bone niche and immune system both in physiological and pathological activities [1]. The real news is to consider bone niche as a dynamic and complex system, undergoing continuous cycles of modeling during growth and remodeling during adulthood, which guarantee adequate mechanical properties over bone growth [2].

Several recent data confirmed that molecular and cellular players involved in the maintenance and accrual of bone mass are many more than originally expected. Specifically, a key player in the maintenance of bone mass in bone pathophysiology is immune system, with immune cells and immune-related factors, such as Interleukins (i.e., IL-6, -11, -17, and -23), Tumor Necrosis Factor (TNF)-a, Nuclear Factor of Activated T-cell, cytoplasmatic-1 (NFATc1) [3-8]. Surprisely, this strict cross talk between the immune system and bone is bidirectional, meaning that bone cells also influence immune cells. Specifically, several works evidenced that osteoblasts contribute to the commitment and differentiation of B-lymphocytes from hematopoietic stem cells [9]. The osteoblasts secretion of Interleukin (IL)-7 and C-X-C motif chemokine Ligand (CXCL)12 leads to the differentiation of cells [10]. On the other hand, many cytokines as IL-1, IL-15 and IL-17f increase osteoblast activity [7]. In addition, osteoclasts have been shown to regulate the immune niche both directly and indirectly through osteoblasts. They increase the mobilization of the immune cells with the secretion of cathepsin $\mathrm{K}$ and the $\mathrm{T}$ Cell, Immune Regulator 1, ATPase, H+ transporting, lysosomal V0 protein A3 (Tcirg1) [11,12]. In addition, several other immune cells and factors promote osteoclastogenesis, such as: neutrophils, sinoviocytes, T-cells, Activated leucocytes, dendritic cells, stimulated stromal cells, NK-cells; M-CSF, TNFa, IL-1a, IL-1b, IL7, IL-8, IL-23, IL-24, IL-34, IL 17, IL 15 [13-21].

The turning point was in 1990, when the Receptor Activator of NF-kB (RANK)/RANK Ligand (RANKL)/Osteoprotegerin (OPG) system was discovered with its critical role in regulating osteoclastogenesis and bone remodeling activity [22]. While the RANK receptor is expressed on the surface of mature osteoclasts and their precursors, RANKL is mainly expressed by stromal cells, osteoblasts, immune cells and released in soluble form in the bone microenvironment. The soluble receptor OPG plays an antagonist role against RANK/RANKL interaction, blocking osteoclast maturation and activity under physiological conditions [23,24]. The equilibrium between RANK and OPG is regulated by activity of several cytokines and systems, as interleukin (IL)-1, IL6, TNF alpha, TNF receptor-associated factors (TRAFs), PI3K, c-Src, Akt/PKB and mTOR [25,26]. Several data evidenced that many of these factors are also involved in immune system regulation. Moreover, RANK/OPG balance plays a fundamental role in immune system activity: it increases lymphocyte 
development in lymph nodes, sustains dendritic cell (DC) maturation and activation, and regulates $\mathrm{T}$ cell-dependent immune response [27-28].

This close link between bone and immune system in physiology is also maintained in pathological conditions. Initial data evidenced that diseases affecting the bone have an immunologic origin, while some immunological and bone disorders, such as rheumatoid arthritis, osteoarthritis and osteoporosis. There is a rise in the existence of these different skeletal diseases, which occur because of defective bone remodeling as a consequence of skewed immune system because of disruption of the homeostatic nexus between bone and immune system and enhanced bone loss [29].

In 2014, Krevvata et al evidenced a correlation between bone niche and cancer cells in acute myeloid leukemia (AML). In this case, osteoblasts can influence the progression of preneoplastic and neoplastic transformations in the myeloid lineage. Specifically, they are able to slow down leukemia progression in several experiments with mice, creating an unfavorable microenvironment for leukemic blast growth. The "bone niche" concept becomes a "niche-induced leukemia" system: for the first time bone niche is evaluated as a dynamic system that include bone, immune and cancer cells [30].

\section{Alterations of bone and immune system in cancer: preclinical data}

Several preclinical data in the last years demonstrated that bone microenvironment and immune system could also play an active role in promoting tumor growth and progression. Bone represents a cancer cells sanctuary against anticancer therapies. The bone niche guarantees an evasion of the immune system and disseminated tumor cells are frequently detected in bone marrow, where the "hematopoietic niche" hides them from anticancer drugs [31]. In addition, tumor cells are able indirectly to reduce their immunogenicity by bypassing tumor immune surveillance mechanism. Although it is mostly unknown how bone homeostasis may shape the immune system, some possible mechanisms begin to be demonstrated. In the bone niche, cancer cells are able to overbalance the RANKL/OPG ratio to osteoclastogenesis, favoring bone resorption and metastases implant. The osteoclastogenesis process leads bone niche to down regulation of immune system pathway, in a vicious circle that enhances tumor bone spread. Several works demonstrated these processes: i) RANK-expressing tumor cells/RANKL activation determines tumor metastatization; ii) T-cell suppression in bone-tumor niche helps bone lysis and tumor cells implantation; iii) T-cell suppression reduces osteoblastogenesis and bone stabilization; iv) Osteoclast factors, such as $\mathrm{TGFb}$, released in the bone marrow inhibits $\mathrm{T}$ cells proliferation and enhanced tumor spread; $\mathrm{v}$ ) Bone niche immune cells, as macrophages, can participate in antitumor responses after anticancer therapy, with elimination of circulating tumor cells and reduction of bone cancer cells implantation;

vi) RANKL/RANK/OPG pathway activation has been identified as a negative prognostic factor in 
cancer development [32-36] [Figure 1].

Prostate cancer is one of tumors which has been analyzed more frequently as regards bone, cancer and immune cell activity. Tumor cells increase the RANKL/OPG ratio indirectly in the bone with the release of different factors such as PTHrP, IL1, IL6 (osteoclast differentiation and survival activity) and directly with osteoclast precursors interaction and co-activation. In addition, cancer cells produce osteoblast differentiation inhibitors such as dickkopf-1 (DKK-1) and activin A [37, 38].

Recently other molecules (e.g. PGE2) are under evaluation as possible regulators in bone resorption and metastatization in prostate cancer and might hopefully become therapeutic targets. Probably tumor cells wedged in bone niche deregulates bone remodeling and manifests as osteolytic lesions that may cause skeletal related events (SREs) (pathologic bone fractures, hypercalcemia) [37].

In addition, multiple myeloma (MM) arises from the clonal expansion of malignant plasma cells within the bone marrow and is often associated with adverse skeletal events. MM is the only skeletal malignancy that is purely lytic and exhibit potent osteolytic capacity through their ability to promote bone resorption by secreting cytokines and chemokines as well as altering the RANKL/OPG ratio within the marrow microenvironment to favor osteoclastogenesis [39]. Schramek et al demonstrated that aberrant RANK/RANKL signaling in mammalian tissues has recently been shown to influence the onset and progression of progestin-mediated breast tumorigenesis. This process is amplified by a synergistic immune cells deregulation, mediated by cytokines such as IL-1, IL-6 and TNF-alpha [40].

For this reason, RANK/RANKL/OPG system became a potential therapeutic target capable of preventing further bone loss, decreasing the number of skeletal-related events and, interestingly, direct antitumor effect through reactivating immune system cells. For the first time we consider a bone target therapy as a possible systemic anticancer effect.

\subsection{Bone targeted drugs have a role in anticancer systemic therapy through immune system activation}

Bisphosphonates were the first drug class that demonstrates the ability to inhibit osteoclast formation, recruitment and adhesion to bone shift the balance towards OPG production by osteoblasts and induce osteoclasts apoptosis [Table 1]. The first effect was to prevent pathological bone resorption with a dramatic skeletal-related events reduction [41].

Clezardin et al in a recent study also demonstrated the existence of direct and indirect antitumor activity. Bisphosphonates reduced the release of bone-derived growth factors and cytokines, counteracting tumor growth and proliferation in the bone niche. In addition, they inhibit 
tumor cell adhesion, invasion, and proliferation, and they induce cancer cells apoptosis (bidirectional bone/cancer cells interaction) [42]. The latest data regarding third generation bisphosphonates evidenced also an indirect antitumor effect via immune system regulation. Zoledronic acid and pamidronate activate $\mathrm{T}$ cells surveillance in bone niche and blood, with antiangiogenic and immune-modulatory mechanism [43].

Breast cancer, MM and prostate cancer are the tumors with zoledronic acid activity which have been studied more [44]. A lot of data confirmed that zoledronic acid prevents aromatase inhibitor induced bone loss, bone metastases, skeletal-related events and reduced survival in patients with metastatic breast cancer [45]. Recent data also evidenced a possible synergistic effect of zoledronic acid with chemotherapy (such as cisplatin) in metastatic triple negative breast cancer, with results in terms of progression free survival and overall survival. Specifically, zoledronic acid mediate its antitumor effects by stimulation of T cells and monocytes plus inhibition of osteoclastmediated bone resorption (bone/cancer/immune cells interaction) [46]. Recent data suggest that bisphosphonates protect the bone and may exert anticancer activity in postmenopausal women with breast cancer treated with adjuvant therapy, hypothesizing that an early use in adjuvant setting could provide the greatest benefits [47]. In addition, in renal cell cancer and hepatocellular carcinoma recent data evidence a systemic activity of bisphosphonates to prolong patient survival and increase quality of life [48-50]

Recently, a new drug blocking RANK/RANKL/OPG pathway exerts bone control and anticancer activity: denosumab, a fully human monoclonal antibody (anti-RANKL), approved for the treatment and prevention of SREs in bone metastases from multiple myeloma, breast and prostate cancer. Better than zoledronic acid, denosumab evidenced many anticancer activities in all studies presented in the last decade: direct osteoclast inhibition, prevention of bone resorption and destruction, reduction of skeletal-related events, immune e system preservation by $\mathrm{B}$ cell/T cell differentiation and dendritic cell survival (both in bone niche and blood) [51]. Initial data evidenced a possible role in systemic tumor control with better progression and overall survival. In addition, in this case the systemic anti-cancer effect seems due to a better immune system regulation, T-cell activation and immunogenic chemokine's increase [52-56]. Current clinical studies are evaluating to a greater extent the effect of denosumab on survival and other biomarkers.

\subsection{Anticancer drugs play effect against bone disruption through immunomodulation}

On the other hand, considering the bone/cancer cells interaction as a bidirectional process, some authors first evaluated a possible converse scenario: a bone disease control using the systemic 
anticancer drugs. Certainly, a better control of systemic cancer disease delays metastases onset, including bone. Recently authors evidenced an indirect bone disease control by new generation antiandrogens, such as the CYP17 inhibitor Abiraterone in prostate cancer [57]. In all clinical studies, Abiraterone demonstrates a better control of systemic disease also thanks to bone resorption control (prolonged radiographic progression free survival), skeletal-related events reduction (time to first skeletal-related event), better quality of life with reduced bone pain. Laboratory data evidenced a possible bone niche control by immune system activation, such as T-cells increase, dendritic cells control and immune-stimulatory cytokines. Detti et al recently also evidenced a possible synergistic activity of abiraterone with bone radiotherapy in bone metastases control in patients with advanced prostate cancer. Radiotherapy in bone niche activates immune system control and exposes immune cells to abiraterone activity. The result is the block of the bone/cancer cells pathway, with osteoclastogenesis reduction and bone stabilization [58]. Other data are necessary to better clarify this bone disease control through immunomodulation.

\section{Conclusions}

Osteoimmunology is a field with broad and general relevance to bone metabolism and in the last years it has changed the therapeutic scenario in cancer bone disease. Until the 2000s, bone niche has been evaluated as a "impenetrable sanctuary" for anti-cancer drugs. Its infiltration by cancer cells has represented a defeat for oncologic treatments, an early progression signal with poor prognosis. After a correct knowledge of bone niche and bone/immune cells pathways system, the osteommunology concept has allowed to develop different potential mechanisms involved in pathologic operation of bone remodeling system. A better understanding of the molecular crosstalk between immune system, skeletal system and cancer cells is needed in order to facilitate the design of possible therapeutic strategies aimed at disrupting this often-pathologic interaction. A bidirectional process between bone and cancer cells could explain a possible both locoregional (bone) and systemic cancer control. We could hypothesize that the immune system represents a "bond", a bridge between bone niche cells and cancer cells. An adequate knowledge of this complex equilibrium can represent a potential therapeutic target to control not only bone metastases, but also systemic cancer pathology. Moreover, after the recent advent of immunotherapy in anticancer drugs scenario, all data could open a future rationale of combined bone, immunologic and targeted therapies in cancer treatment.

Table 1.

Activity data of bisphosphonates and denosumab in cancer type 


\begin{tabular}{ll}
\hline & Lung cancer \\
& Renal cell carcinoma \\
& Prostate cancer \\
\hline Denosumab & Multiple myeloma \\
& Breast cancer \\
& Lung cancer \\
& Prostate cancer \\
& Hepatocellular carcinoma \\
& Hewing Sarcoma \\
\hline
\end{tabular}

Figure 1.

Bone, immune and cancer cells in cancer development: bone resorption and osteoclastogenesis

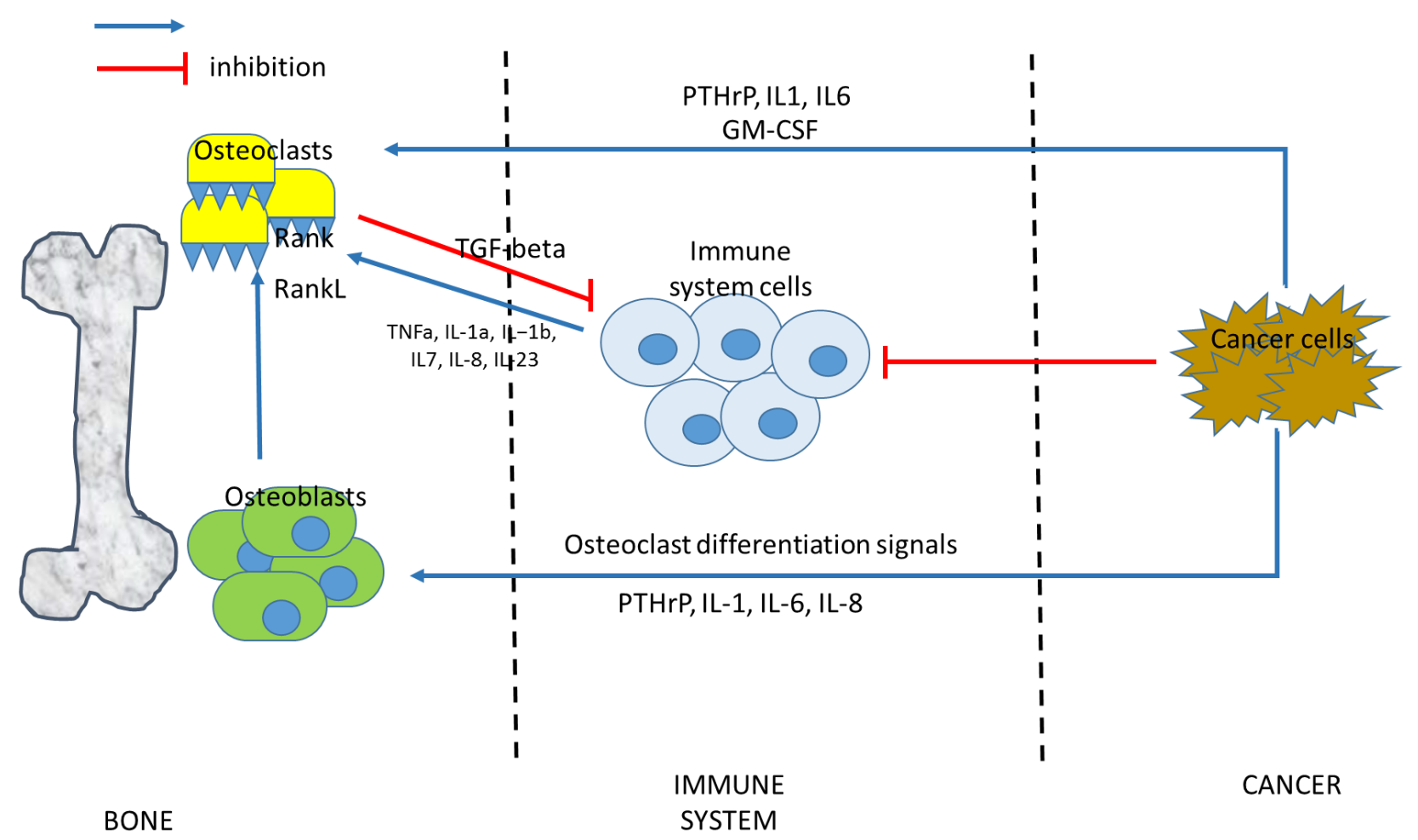

\section{References.}

1. Arron, J,R,; Choi, Y. Bone versus immune system. Nature 2000, 408, 535-6. 
2. Cappariello, A.; Maurizi, A.; Veeriah, V.; Teti, A. The great beauty of osteoclast. Arch Biochem Biophys 2014, 561, 13-21.

3. Mori, G.; D'Amelio, P.; Faccio, R.; Brunetti, G. The Interplay between the bone and the immune system. Clin Dev Immunol 2013, 720504.

4. De Benedetti, F.; Rucci, N.; Del Fattore, A.; Peruzzi, B.; Paro, R.; Longo, M.; et al. Impaired skeletal development in interleukin-6-transgenic mice: a model for the impact of chronic inflammation on the growing skeletal system. Arthritis Rheum 2006, 54, 3551-63.

5. Nam, D.; Mau, E.; Wang, Y.; Wright, D.; Silkstone, D.; Whetstone, H.; et al. T-lymphocytes enable osteoblast maturation via IL-17F during early phase of fracture repair. PLoS ONE 2012, 7, e40044.

6. Basile, D.; Garattini, S.K.; Bonotto, M.; Ongaro, E.; Casagrande, M.; Cattaneo, M.; et al. Immunotherapy for colorectal cancer: where are we heading? Expert Opin Biol Ther 2017, 17, 709721.

7. Takeda, H.; Kikuchi, T.; Soboku, K.; Okabe, I.; Mizutani, H.; Mitani, A, et al. Effect of IL15 and natural killer cells on osteoclasts and osteoblasts in a mouse coculture. Inflammation 2014, 37, 657-69.

8. Guihard, P.; Boutet, M.A.; Brounais, B.; David, E.; Brion, R.; Delecrin, J.; et al. Induction of osteogenesis in mesenchymal stem cells by activated monocytes/macrophages depends on oncostatin M signaling. Stem Cells 2012, 30, 762-72.

9. Zhu, J.; Garrett, R.; Jung, Y.; Kim, N.; Wang, J.; Joe, G.J.; et al. Osteoblasts support Blymphocyte commitment and differentiation from hematopoietic stem cells. Blood 2007, 109, 3706-12.

10. Miller, J.P.; Izon, D.; De Muth, W.; Gerstein, R.; Bhandoola, A.; Allman, D. The earliest step in B lineage differentiation from common lymphoid progenitors is critically dependent upon interleukin 7. J Exp Med 2002, 196, 705-11.

11. Seifert, M.F.; Marks, S.C. Jr. Morphological evidence of reduced bone resorption in the osteosclerotic (oc) mouse. Am J Anat, 1985, 172, 141-53.

12. Mansour, A.; Abou-Ezzi, G.; Sitnicka, E.; Jacobsen, S.E.; Wakkach, A.; Blin-Wakkach, C. Osteoclasts promote the formation of hematopoietic stem cell niches in the bone marrow. $J$ Exp Med 2012, 209, 537-49.

13. Kitaura, H.; Zhou, P.; Kim, H.J.; Novack, D.V.; Ross, F.P.; Teitelbaum, S.L. MCSF mediates TNF-induced inflammatory osteolysis. J Clin Invest 2005, 115, 3418-27.

14. Jarry, C.R.; Durante, P.M.; Freitas, F.F.; de Macedo, C.G.; Clemente-Napimoga, J.T.; SabaChujfi, E.; et al. Secreted osteoclastogenic factor of activated T cells (SOFAT), a novel osteoclast activator, in chronic periodontitis. Hum Immunol 2013, 74, 861866.

15. Weitzmann, M.N.; Cenci, S.; Rifas, L.; Brown, C.; Pacifici, R. Interleukin-7 stimulates osteoclast formation by up-regulating the T-cell production of soluble osteoclastogenic cytokines. Blood 2000, 96, 1873-8.

16. Amarasekara, D.S.; Yun, H.; Kim, S.; Lee, N; Kim, H.; Rho, J. Regulation of osteoclast differentiation by cytokine networks. Immune Netw 2018, 18, e8.

17. Kudo, O.; Sabokbar, A.; Pocock, A.; Itonaga, I.; Fujikawa, Y.; Athanasou, N.A. Interleukin-6 and interleukin-11 support human osteoclast formation by a RANKL-independent mechanism. Bone 2003, 32, 1-7.

18. Chen, L.; Wei, X.Q.; Evans, B.; Jiang, W.; Aeschlimann, D. IL-23 promotes osteoclast formation by up-regulation of receptor activator of NF-kappaB (RANK) expression in myeloid precursor cells. Eur J Immunol 2008, 38, 2845-54.

19. Raisz, L.G. Prostaglandins and bone: physiology and pathophysiology. Osteoarthritis Cartilage 1999, 7, 419-21.

20. Moon, Y.M.; Yoon, B.Y.; Her, Y.M.; Oh, H.J.; Lee, J.S.; Kim, K.W.; et al. IL-32 and IL-17 interact and have the potential to aggravate osteoclastogenesis in rheumatoid arthritis. Arthritis Res Ther 2012, 14, R246.

21. Yoshitake, F; Itoh, S.; Narita, H.; Ishihara, K.; Ebisu, S. Interleukin-6 directly inhibits 
osteoclast differentiation by suppressing receptor activator of NF-kappaB signaling pathways. $J$ Biol Chem, 2008, 283, 11535-40.

22. Boyce, B.F.; Xing, L. The RANKL/RANK/OPG pathway. Curr Osteoporosis Rep 2007, 5, 98-104.

23. Khosla, S. Minireview: the OPG/RANKL/RANK system. Endocrinology 2001, 142, 5050-5. 24. Lee, N.K.; Choi, Y.G.; Baik, J.Y.; Han, S.Y.; Jeong, D.W.; Bae, Y.S.; et al. A crucial role for reactive oxygen species in RANKL-induced osteoclast differentiation. Blood 2005, 106, 852-9.

25. Lorenzo, J. Interactions between immune and bone cells: new insights with many remaining questions. J Clin Investig 2000, 106, 749-52.

26. Theill, L.E.; Boyle, W.J.; Penninger, J.M. RANK-L and RANK: T cells, bone loss, and mammalian evolution. Annu Rev Immunol 2002, 20, 795-823.

27. Calvi, L.M.; Adams, G.B.; Weibrecht, K.W.; Weber, J.M.; Olson, D.P.; Knight, M.C.; et al. Osteoblastic cells regulate the haematopoietic stem cell niche. Nature 2003, 425, 841-6.

28. Loser, K.; Mehling, A.; Loeser, S.; Apelt, J.; Kuhn, A.; Grabbe, S.; et al. Epidermal RANKL controls regulatory T-cell numbers via activation of dendritic cells. Nat Med 2006, 12, 1372-9.

29. Takayanagi, H. Osteoimmunology: shared mechanisms and crosstalk between the immune and bone systems. Nat Rev Immunol 2007, 7, 292-304.

30. Krevvata, M.; Silva, B.C.; Manavalan, J.S.; Galan-Diez, M.; Kode, A.; Matthews, B.G. et al. Inhibition of leukemia cell engraftment and disease progression in mice by osteoblasts. Blood 2014, 124, 2834-46.

31. Terpos, E.; Dimopoulos, M.A. Interaction between the skeletal and immune systems in cancer: mechanisms and clinical implications. Cancer Immunol Immunother 2011, 60, 305-17.

32. Schreiber, R.D.; Old, L.J.; Smyth, M.J. Cancer immunoediting: integrating immunity's roles in cancer suppression and promotion. Science 2011, 331, 1565-70.

33. Bidwell, B.N.; Slaney, C.Y.; Withana, N.P.; Forster, S.; Cao, Y.; Loi, S.; et al. Silencing of Irf7 pathways in breast cancer cells promotes bone metastasis through immune escape. Nat Med 2012, 18, 1224-31.

34. Morony, S.; Capparelli, C.; Sarosi, I.; Lacey, D.L.; Dunstan, C.R.; Kostenuik, P.J. Osteoprotegerin inhibits osteolysis and decreases skeletal tumor burden in syngeneic and nude mouse models of experimental bone metastasis. Cancer Res 2001, 61, 4432-6.

35. Roodman, G.D.; Dougall, W.C. RANK ligand as a therapeutic target for bone metastases and multiple myeloma. Cancer Treat Rev 2008, 34, 92-101.

36. Jones, D.H.; Nakashima, T.; Sanchez, O.H.; Kozieradzki, I.; Komarova, S.V.; Sarosi, I.; et al. Regulation of cancer cell migration and bone metastasis by RANKL. Nature 2006, 440, 692-6.

37. Di Francesco, S.; Castellan, P.; Manco, R.; Tenaglia, R.L. Reciprocal cross-talk between Prostaglandin E2 and bone in prostate cancer: a current review. Cent Eur J Urol 2011, 64, 201-4.

38. Wong, S.K.; Mohamad, N.V.; Giaze, T.R.; Chin, K.Y.; Mohamed, N.; Ima-Nirwana, S. Prostate Cancer and Bone Metastases: The Underlying Mechanisms. Int J Mol Sci 2019, 27, 20.

39. Colucci, S.; Brunetti, G.; Rizzi, R.; Zonno, A.; Mori, G.; Colaianni, G.; et al. T cells support osteoclastogenesis in an in vitro model derived from human multiple myeloma bone disease: the role of the OPG/TRAIL interaction. Blood 2004, 104, 3722-30.

40. Schramek, D.; Leibbrandt, A.; Sigl, V.; Kenner, L.; Pospisilik, J.A.; Lee, H.J.; et al. Osteoclast differentiation factor RANKL controls development of progestindriven mammary cancer. Nature 2010, 468, 98-102.

41. Russell, R.G. Bisphosphonates: from bench to bedside. Ann N Y Acad Sci 2006, 1068, 367401.

42. Clezardin, P. Potential anticancer properties of bisphosphonates: insights from preclinical studies. Anti-Cancer Agents Med Chem 2012, 12, 102-13.

43. McCloskey, E.V.; Dunn, J.A.; Kanis, J.A.; MacLennan, I.C.; Drayson, M.T. Long-term follow-up of a prospective, double-blind, placebo-controlled randomized trial of clodronate in multiple myeloma. Br J Haematol 2001, 113, 1035-43.

44. Palmieri, C.; Fullarton, J.R.; Brown, J. Comparative efficacy of bisphosphonates in 
metastatic breast and prostate cancer and multiple myeloma: a mixedtreatment meta-analysis. Clin Cancer Res 2013, 19, 6863-72.

45. Anagha, P.P.; Sen, S. The efficacy of bisphosphonates in preventing aromatase inhibitor induced bone loss for postmenopausal women with early breast cancer: a systematic review and meta-analysis. J Oncol 2014, 2014, 625060.

46. Ibrahim, T.; Liverani, C.; Mercatali, L.; Sacanna, E.; Zanoni, M.; Fabbri, F.; et al. Cisplatin in combination with zoledronic acid: a synergistic effect in triple-negative breast cancer cell lines. Int J Oncol 2013, 42, 1263-70.

47. Valachis, A.; Polyzos, N.P.; Coleman, R.E.; Gnant, M.; Eidtmann, H.; Brufsky, A.M.; et al. Adjuvant therapy with zoledronic acid in patients with breast cancer: a systematic review and metaanalysis. Oncologist 2013, 18, 353-61.

48. Santini, D.; Procopio, G.; Porta, C.; Ibrahim, T.; Barni, S.; Mazzara, C.; et al. Natural history of malignant bone disease in renal cancer: final results of an Italian bone metastasis survey. PLoS One 2013, 8, e83026.

49. Santoni, M.; Conti, A.; Procopio, G.; Porta, C.; Ibrahim, T.; Barni, S.; et al. Bone metastases in patients with metastatic renal cell carcinoma: are they always associated with poor prognosis? $J$ Exp Clin Cancer Res 2015, 5, 34:10.

50. Casadei Gardini, A.; Scarpi, E.; Faloppi, L.; Scartozzi, M.; Silvestris, N.; Santini, D.; et al. Immune inflammation indicators and implication for immune modulation strategies in advanced hepatocellular carcinoma patients receiving sorafenib. Oncotarget 2016, 7, 67142-67149.

51. Goessl, C.; Katz, L.; Dougall, W.C.; Kostenuik, P.J.; Zoog, H.B.; Braun, A.; et al. The development of denosumab for the treatment of diseases of bone loss and cancer-induced bone destruction. Ann N Y Acad Sci 2012, 1263, 29-40.

52. Rolfo, C.; Raez, L.E.; Russo, A.; Reguart, N.; Campelo, R.G.; Bronte, G.; et al. Molecular target therapy for bone metastasis: starting a new era with denosumab, a RANKL inhibitor. Expert Opin Biol Ther 2014, 14, 15-26.

53. Lipton, A.; Fizazi, K.; Stopeck, A.T.; Henry, D.H.; Smith, M.R.; Shore, N.; et al. Effect of denosumab versus zoledronic acid in preventing skeletal-related events in patients with bone metastases by baseline characteristics. Eur J Cancer 2016, 53, 75-83.

54. Stopeck, A.T.; Fizazi, K.; Body, J.J.; Brown, J.E.; Carducci, M.; Diel, I.; et al. Safety of long-term denosumab therapy: results from the open label extension phase of two phase 3 studies in patients with metastatic breast and prostate cancer. Support Care Cancer 2016, 24, 447-455.

55. Galvano, A.; Scaturro, D.; Badalamenti, G.; Incorvaia, L.; Rizzo, S.; Castellana, L.; et al. Denosumab for bone health in prostate and breast cancer patients receiving endocrine therapy? A systematic review and a meta-analysis of randomized trials. J Bone Oncol 2019, 18, 100252.

56. Dionísio, M.R.; Mansinho, A.; Abreu, C.; Cavaco-Silva, J.; Casimiro, S.; Costa, L. Clinical and translational pharmacology of drugs for the prevention and treatment of bone metastases and cancer-induced bone loss. Br J Clin Pharmacol 2019, 85, 1114-1124.

57. Rizzo, S.; Galvano, A.; Pantano, F.; Iuliani, M.; Vincenzi, B.; Passiglia, F.; et al. The effects of enzalutamide and abiraterone on skeletal related events and bone radiological progression free survival in castration resistant prostate cancer patients: An indirect comparison of randomized controlled trials. Crit Rev Oncol Hematol 2017, 120, 227-233.

58. Detti, B.; D'Angelillo, R.M.; Ingrosso, G.; Olmetto, E.; Francolini, G.; Triggiani, L.; et al. Combining Abiraterone and Radiotherapy in Prostate Cancer Patients Who Progressed During Abiraterone Therapy. Anticancer Res 2017, 37, 3717-3722. 